\title{
Plasma calcium fractions and the protein-binding of calcium in normal subjects and in patients with hypercalcaemia and hypocalcaemia
}

\author{
M. R. WILLS AND M. R. LEWIN \\ From the Department of Chemical Pathology, Royal Free Hospital, Lawn Road, London
}

SYNOPSIS A study is reported of the estimation of plasma calcium fractions and the calcium-binding affinity of plasma proteins in a total sample of 59 people, which included 29 normal subjects and of 30 patients with either hypercalcaemia or hypocalcaemia. It was demonstrated that when the sample

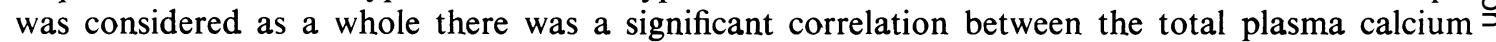
concentration and the ultrafiltrable, ionized, and protein-bound calcium fractions and between $\vec{\square}$ the ultrafiltrable and ionized fractions. We have also demonstrated that in patients with either ${ }^{\mathbb{D}}$ hypercalcaemia or hypocalcaemia, including acidotic uraemics, the calcium-binding affinity of the $\stackrel{\Phi}{\rightrightarrows}$ plasma proteins did not differ significantly from that in normal subjects. A significant correlation was also found between the total plasma calcium concentration and the ultrafiltrable, ionized and protein-bound calcium fractions when the normal subjects and the groups of patients with hypercalcaemia and hypocalcaemia due to chronic renal failure were considered as separate groups. The group of patients with hypercalcaemia included patients both with hyperparathyroidism and with hypercalcaemia due to other causes.

Calcium is present in blood plasma in three distinct fractions which are in equilibrium with one another, ie, the ionized and the complexed calcium which together comprise the diffusible or ultrafiltrable fraction, and the non-diffusible calcium which is bound to the plasma proteins. The ionized calcium is considered to be the physiologically active fraction (McLean and Hastings, 1935) under the control of the parathyroid glands (McLean, Barnes, and Hastings, 1934). In this now classical concept (Neuman and Neuman, 1958; Fourman and Royer, 1968) of the state and control of calcium fractions in plasma, one would expect the measurement of these variables to be of value in the diagnosis of disturbances of calcium homeostasis with either hypocalcaemia or hypercalcaemia, in the latter case with particular reference to parathyroid gland overactivity. Many workers, using varying techniques, have estimated plasma calcium fractions in disease states with conflicting results, some suggesting that the binding affinity of plasma proteins for calcium may be abnormal in certain diseases (vide infra).

The amount of calcium which is bound by the plasma proteins is both $p \mathrm{H}$ - and temperaturedependent (Toribara, Terepka, and Dewey, 1957; Loken, Havel, Gordan, and Whittington, 1960), Received for publication 13 September 1971. but the calcium proteinate dissociation constant $\stackrel{\mathbb{D}}{\circ}$ has never been calculated from complete data deter- $\overrightarrow{\vec{O}}$ mined under physiological conditions of both $p \mathrm{H} 3$ and temperature. Many workers have calculated the binding constant from a knowledge of the? diffusible calcium only, on the assumption that it closely approximates the level of ionized calcium? (Hopkins, Howard, and Eisenberg, 1952; Loken et al, 1960; Walser, 1962). Others have calculated the binding constant from variables determined at room temperature (Lloyd, Rose, and Smeenk, 1962; Dale and Kellerman, 1967). The study reported here was 을 undertaken to measure plasma calcium fractions $\frac{D}{0}$ and to determine the calcium proteinate dissociation constant from complete data, measured under $N$ physiological conditions of $p \mathrm{H}$ and temperature, in both normal subjects and in patients with either hypercalcaemia or hypocalcaemia over a wide range $\omega$ of total plasma calcium concentrations. Plasma calcium fractions were measured as far as possibleco under in vivo conditions.

\section{Subjects Studied}

GROUP I: NORMAL SUBJECTS

Twenty-nine normal subjects were studied, all $\stackrel{\AA}{\perp}$ healthy laboratory workers with no evidence of a? 
disturbance of calcium homeostasis. The group comprised 15 males and 14 females; ages ranged from 17 to 59 years.

\section{GROUP II: HYPERCALCAEMIA}

Nine patients were studied, five with hyperparathyroidism and four with metastatic carcinoma. In the patients reported here and others referred to from the literature those termed 'hyperparathyroidism' represent cases of proven primary hyperparathyroidism unless otherwise stated.

\section{GROUP III: HYPOCALCAEMIA}

A total of 21 patients with hypocalcaemia due either to chronic renal failure or various other causes have been studied. In discussing this subgroup we refer to those with hypocalcaemia due to renal failure as 'renal hypocalcaemia' and to the others as 'non-renal hypocalcaemia'.

\section{IIIa Non-renal hypocalcaemia}

Twelve patients were studied with hypocalcaemia due to various non-renal causes; in one patient this was due to hypoparathyroidism after surgery, in the others to either gastrointestinal or hepatic disease.

\section{IIIb Renal hypocalcaemia}

Nine patients with end-stage chronic renal failure were studied; none had undergone dialysis treatment at the time of study. All were being managed on a medical (low protein) regimen, and their blood urea concentrations ranged from 54 to $300 \mathrm{mg}$ per $100 \mathrm{ml}$.

1 All estimations were performed on venous blood specimens collected by one of us. The samples (approximately $30 \mathrm{ml}$ ) were collected anaerobically, without stasis, into a $30 \mathrm{ml}$ plastic syringe the dead space of which was filled with heparin (Evans heparin 1,000 units per $\mathrm{ml}$ ), giving a blood concentration of about 10 units $/ \mathrm{ml}$.

2 Total and ultrafiltrable plasma calcium concentrations were estimated by the fluorimetric method of Lewin, Wills, and Baron (1969) after anaerobic separation under liquid paraffin. The within-batch precision of the method was calculated from 20 replicate estimations performed on a single plasma sample. The coefficient of variation was found to be $0.57 \%$.

3 A plasma ultrafiltrate was obtained using a negative mercury pressure method $(60 \mathrm{~mm} \mathrm{Hg}$ ) under anaerobic conditions at $37^{\circ} \mathrm{C}$. Immediately after collection of the heparinized blood samples, the plasma and red cell phases were separated by centrifugation under liquid paraffin. The ultrafiltration apparatus used was based upon the LKB ultrafilter (LKB 6300A, Stockholm, Sweden), and was constructed to allow for the ultrafiltration of 2 to $3 \mathrm{ml}$ volumes of plasma. Before the final assembly of the apparatus and during the ultrafiltration procedure the outer compartment above the oil was flushed through with $5 \% \mathrm{CO}_{2}$ in oxygen saturated with water vapour at $37^{\circ} \mathrm{C}$. The whole procedure was carried out in a $37^{\circ} \mathrm{C}$ water bath. With this apparatus we were able to obtain approximately $0.3 \mathrm{ml}$ of ultrafiltrate in one hour. Over that time period the loss by evaporation was not significant $(P>0.9)$ nor was there a significant change in the plasma $p \mathrm{H}$ $(\mathrm{P}>0 \cdot 2)$.

4 Plasma ionized calcium concentration was measured by a two-wavelength, spectrophotometric murexide method based upon that of Lumb (1963). Immediately after collection of the heparinized blood samples the plasma was separated by centrifugation under liquid paraffin; $2.0 \mathrm{ml}$ of plasma was then transferred anaerobically to a cuvette containing $20 \mu \mathrm{l}$ of aqueous murexide (recrystallized) solution $(8.0 \mathrm{mg}$ per $10.0 \mathrm{ml})$. The cuvette was then sealed, placed in a $37^{\circ} \mathrm{C}$ water bath for five minutes and then transferred to a thermostatted cuvette holder $\left(37^{\circ} \mathrm{C}\right)$ in a Hilger and Watts spectrophotometer (H700.308). Absorbance was measured at 480 and $520 \mathrm{~nm}$ against a plasma blank $(2.0 \mathrm{ml}$ plasma with $20 \mu \mathrm{l}$ water), and the absorbance ratio calculated. These two wavelengths represent the absorbance maxima respectively of the calcium murexide complex and the murexide solution (Ettori and Scoggan, 1961). Four buffered sets of standard calcium solutions ranging from 0 to $10.0 \mathrm{mg}$ per $100 \mathrm{ml}$ of calcium were prepared, sodium chloride being added to bring the final ionic strength to $0.15 \mathrm{M}$. Each set of standards was buffered with tris to give final $p H$ values of $7 \cdot 23,7 \cdot 32,7 \cdot 36$, and $7 \cdot 42$. The plasma ionized calcium concentration was then determined from a calibration curve constructed, at the same time, from the nearest set of buffered standards with a $p H$ comparable to that of the original blood sample. The within-batch precision of the method was calculated from 20 estimations performed on a single pooled plasma sample and the coefficient of variation was found to be $2.11 \%$. The recovery of the method was determined by adding calcium to a standard $2.0 \mathrm{mg}$ per $100 \mathrm{ml}$ calcium solution to give final calcium concentrations of $3 \cdot 0,4 \cdot 0,5 \cdot 0,6 \cdot 0$, and $7.0 \mathrm{mg}$ per $100 \mathrm{ml}$. At each of these final concentrations three estimations of recovery were determined. Over the whole range the results varied between 97.5 and $101 \%$, with a mean recovery of $99.4 \%$. 
While the work reported here was in progress Robertson and Peacock (1968) reported a method for the potentiometric measurement of ionized calcium concentrations in a plasma ultrafiltrate using a specific divalent cation electrode. The plasma ultrafiltrate was prepared under controlled conditions, similar to those reported here, which they considered to "duplicate the conditions existing in vivo especially with respect to $p \mathrm{H}$, carbon dioxide tension $\left(\mathrm{PCO}_{2}\right)$, and temperature'. Using this technique they found that in 21 normal subjects when the concentration of the ionized calcium fraction was expressed as a percentage of the total the values (mean \pm SD) were $50.8 \pm 2.2$ which agrees with the value of $50.86 \pm 1.82$ in the study reported here, and validates the method used for the estimation of ionized calcium.

5 pH measurements were made on whole blood at $37^{\circ} \mathrm{C}$ with a capillary glass electrode (EIL ${ }^{1}$ model SHH 33) with a Radiometer $p H$ meter (model 27).

$6 \mathrm{PCO}_{2}$ measurements were made on whole blood using a Severinghaus $\mathrm{PCO}_{2}$ electrode (EIL ${ }^{1}$ model 9987 100).

7 Plasma bicarbonate concentrations were measured, as total $\mathrm{CO}_{2}$, on a Natelson microgasometer (Natelson, 1951).

8 Total protein and the albumin and globulin fractions were estimated by the biuret method of Wolfson, Cohn, Calvary, and Ichiba (1948).

\section{Calculations}

In order to relate total, ultrafiltrable, and ionized calcium concentrations and derive the proteinbound and complexed fractions we considered it essential to express the results in the same unit and consequently have reported our results in terms of $\mathrm{mg}$ per $100 \mathrm{ml}$ plasma water. In keeping with modern nomenclature the results should, perhaps, have been expressed in mmol per $\mathrm{kg}$ plasma water. As in the previous reports in the literature the results were expressed in $\mathrm{mg}$ we have retained this terminology for the purposes of this paper.

Plasma water content was determined from the formula of McLean and Hastings (1935):

Water in $100 \mathrm{ml}$ plasma $=(100-1)-0.75 \mathrm{P}$

where 0.75 was the partial specific volume of the plasma proteins; $\mathbf{P}$ was the total protein concentration in $\mathrm{g}$ per $100 \mathrm{ml}$ plasma; and $1 \%$ was the volume assumed to be occupied by solids other than proteins. The factor derived from this formula was used to correct the estimated total and ionized calcium concentrations from $\mathrm{mg}$ per $100 \mathrm{ml}$ plasma to $\mathrm{mg}$ per $100 \mathrm{ml}$ plasma water.

IElectronic Instruments Ltd., Richmond, Surrey.

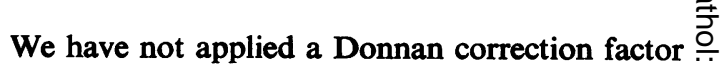
to the measured concentration of calcium in the $\vec{F}$ ultrafiltrate. Robertson and Peacock (1968) in their $\stackrel{\text { ? }}{\rightarrow}$ studies considered that since Donnan equilibria exist 'in vivo' it is unnecessary to make such a correction. In any case Pedersen (1969) from his studies concluded that the Donnan factor under $\triangle$ these conditions of ultrafiltration is negligible.

We have calculated the protein-bound and complexed calcium fractions as the difference between the total and ultrafiltrable and the ultrafiltrable and ionized calcium concentrations respectively.

To determine the calcium proteinate dissociation constant the mass law equation of McLean and Hastings (1935) was used:

$$
\frac{\left[\mathrm{Ca}^{++}\right]\left[\text {Prot }^{-}\right]}{[\text {CaProt }]}=\mathrm{K}_{\mathrm{CaProt}}
$$

$\left[\mathrm{Ca}^{++}\right] \doteq$ concentration of ionized calcium in $\mathrm{mmol} / \mathrm{kg}$ plasma water; the latter was obtained by dividing by 4 the concentration in $\mathrm{mg} / 100 \mathrm{ml}$ plasma water.

[CaProt $]=$ concentration of protein-bound calcium in $\mathrm{mmol} / \mathrm{kg}$ plasma water, derived from the difference between the total and ultrafiltrable calcium concentrations.

[Prot ${ }^{=}$] = concentration of 'free' protein anions.

The concentration of total cation-binding proteins (TCBP) was calculated from the individual plasma albumin and total globulin concentrations and blood $p \mathrm{H}$, using the formula of van Slyke, Hastings, Hiller, and Sendroy (1928):

TCBP $=$

$\frac{0.78 \times \mathrm{Alb} \times(p \mathrm{H}-5.16)+0.48 \times \mathrm{Glob} \times(p \mathrm{H}-4.89)}{2}$ $\mathrm{mmol} / \mathrm{kg}$ plasma water

(Alb and Glob are the albumin and total globulin concentrations converted to $\mathrm{g}$ protein nitrogen $/ \mathbf{k g}$ plasma water as proposed by McLean and Hastings, 1935.)

Prot ${ }^{-}$was then derived from:

$$
\text { [Prot } \left.{ }^{-}\right]=\text {[TCBP] }-(\text { CaProt }]
$$

$\mathrm{K}_{\mathrm{CaProt}}=$ calcium proteinate dissociation constant.

Results

The results of plasma calcium fractions and $\mathrm{K}_{\mathrm{CaProt}}$ values for the normal subjects are shown in Table I. The values (mean \pm SD) for the ultrafiltrable, ionized, protein-bound, and complexed calcium fractions when expressed as a percentage of the total plasma calcium concentration in plasma water were $57.29 \pm 1 \cdot 70,50.86 \pm 1 \cdot 82,42 \cdot 71 \pm 1 \cdot 70$, and 6.44 $\pm 2 \cdot 30$ respectively.

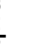




\begin{tabular}{|c|c|c|c|c|c|c|c|c|}
\hline & \multicolumn{2}{|c|}{ Calcium (mg/100 ml plasma) } & \multicolumn{5}{|c|}{ Calcium ( $\mathrm{mg}$ per $100 \mathrm{ml}$ plasma water) } & \multirow[t]{2}{*}{$K_{\text {CaProt }}$} \\
\hline & Total & Ionized & Total & Ultrafiltrable & Ionized & Protein-bound & Complexed & \\
\hline $\begin{array}{l}\text { Mean } \\
\pm S D\end{array}$ & $\begin{array}{r}9.41 \\
\pm 0.29\end{array}$ & $\begin{array}{r}4 \cdot 80 \\
+0.24\end{array}$ & $\begin{array}{r}10.06 \\
\pm 0.32\end{array}$ & $\begin{array}{r}5.76 \\
\pm 0.25\end{array}$ & $\begin{array}{r}5.11 \\
\pm 0.27\end{array}$ & $\begin{array}{r}4 \cdot 26 \\
\pm 0.24\end{array}$ & $\begin{array}{r}0.64 \\
\pm 0.22\end{array}$ & $\begin{array}{r}0.01017 \\
\pm 0.00110\end{array}$ \\
\hline
\end{tabular}

Table I Plasma calcium fractions and $K_{\text {CaProt }}$ values for the 29 normal subjects

\begin{tabular}{|c|c|c|c|c|c|c|c|c|c|c|c|}
\hline \multirow[t]{2}{*}{ Patient } & \multicolumn{2}{|c|}{$\begin{array}{l}\text { Calcium } \\
(\mathrm{mg} / 100 \mathrm{ml} \text { plasma })\end{array}$} & \multicolumn{5}{|c|}{ Calcium (mg per $100 \mathrm{ml}$ plasma water) } & \multirow[t]{2}{*}{$\boldsymbol{K}_{\text {CaProt }}$} & \multicolumn{3}{|c|}{ Protein ( $\mathrm{g}$ per $100 \mathrm{ml}$ plasma) } \\
\hline & Total & Ionized & Total & $\begin{array}{l}\text { Ultra- } \\
\text { filtrable }\end{array}$ & Ionized & $\begin{array}{l}\text { Protein- } \\
\text { bound }\end{array}$ & Complexed & & Total & Albumin & Globulin \\
\hline $\begin{array}{l}1 \\
2 \\
3 \\
4 \\
5 \\
6 \\
7 \\
8 \\
9\end{array}$ & $\begin{array}{l}11.0 \\
11.5 \\
12.0 \\
12.2 \\
13.9 \\
11.5 \\
11.2 \\
10.9 \\
13.2\end{array}$ & $\begin{array}{l}5 \cdot 5 \\
6 \cdot 7 \\
6 \cdot 5 \\
6 \cdot 5 \\
7 \cdot 2 \\
6 \cdot 2 \\
6 \cdot 0 \\
5 \cdot 8 \\
7 \cdot 9\end{array}$ & $\begin{array}{l}11.7 \\
12.3 \\
12.8 \\
13.0 \\
14.8 \\
12.1 \\
12.0 \\
11.7 \\
14.2\end{array}$ & $\begin{array}{l}5 \cdot 9 \\
8 \cdot 1 \\
7 \cdot 4 \\
7 \cdot 7 \\
7 \cdot 9 \\
7 \cdot 4 \\
7 \cdot 4 \\
6 \cdot 6 \\
8 \cdot 7\end{array}$ & $\begin{array}{l}5 \cdot 8 \\
7 \cdot 1 \\
7 \cdot 0 \\
6 \cdot 9 \\
7 \cdot 6 \\
6 \cdot 5 \\
6 \cdot 4 \\
6 \cdot 2 \\
8 \cdot 5\end{array}$ & $\begin{array}{l}5 \cdot 8 \\
4 \cdot 2 \\
5 \cdot 4 \\
5 \cdot 3 \\
6 \cdot 9 \\
4 \cdot 7 \\
4 \cdot 6 \\
5 \cdot 1 \\
5 \cdot 5\end{array}$ & $\begin{array}{l}0.1 \\
1.0 \\
0.4 \\
0.8 \\
0.3 \\
0.9 \\
1.0 \\
0.4 \\
0.2\end{array}$ & $\begin{array}{l}0.00586 \\
0.01341 \\
0.01062 \\
0.00995 \\
0.00730 \\
0.00941 \\
0.01191 \\
0.01058 \\
0.01331\end{array}$ & $\begin{array}{l}6 \cdot 7 \\
7 \cdot 0 \\
7 \cdot 9 \\
7 \cdot 2 \\
6 \cdot 8 \\
6 \cdot 0 \\
7 \cdot 2 \\
8 \cdot 1 \\
8 \cdot 5\end{array}$ & $\begin{array}{l}4 \cdot 3 \\
4 \cdot 6 \\
5 \cdot 1 \\
4 \cdot 6 \\
4 \cdot 3 \\
3.9 \\
4 \cdot 8 \\
3 \cdot 0 \\
2 \cdot 9\end{array}$ & $\begin{array}{l}2 \cdot 4 \\
2 \cdot 4 \\
2 \cdot 8 \\
2 \cdot 6 \\
2 \cdot 5 \\
2 \cdot 1 \\
2 \cdot 4 \\
5 \cdot 1 \\
5 \cdot 6\end{array}$ \\
\hline $\begin{array}{l}\text { Mean } \\
\pm \text { SD }\end{array}$ & $\begin{array}{r}11.93 \\
\pm 1.02\end{array}$ & $\begin{array}{r}6.48 \\
\pm 0.73\end{array}$ & $\begin{array}{l}12 \cdot 74 \\
\pm 1 \cdot 11\end{array}$ & $\begin{array}{r}7.44 \\
\pm 0.83\end{array}$ & $\begin{array}{r}6.88 \\
\pm 0.81\end{array}$ & $\begin{array}{r}5 \cdot 28 \\
\pm 0.79\end{array}$ & $\begin{array}{r}0.57 \\
\pm 0.36\end{array}$ & $\begin{array}{r}0.01026 \\
\pm 0.00253\end{array}$ & $\begin{array}{r}7 \cdot 27 \\
\pm 0.78\end{array}$ & $\begin{array}{r}4 \cdot 17 \\
\pm 0.77\end{array}$ & $\begin{array}{r}3 \cdot 10 \\
\pm 1 \cdot 30\end{array}$ \\
\hline $\begin{array}{l}\text { Patients } 1-5 \\
\text { Mean } \\
\pm \text { SD }\end{array}$ & $\begin{array}{r}12 \cdot 12 \\
+1 \cdot 10\end{array}$ & $\begin{array}{r}6.48 \\
\pm 0.62\end{array}$ & $\begin{aligned} & 12 \cdot 92 \\
\pm & 1 \cdot 16\end{aligned}$ & $\begin{array}{r}7.40 \\
\pm 0.89\end{array}$ & $\begin{array}{r}6.89 \\
\pm 0.66\end{array}$ & $\begin{array}{r}5.52 \\
\pm 0.97\end{array}$ & $\begin{array}{r}0.52 \\
\pm 0.37\end{array}$ & $\begin{array}{r}0.00943 \\
\pm 0.00295\end{array}$ & $\begin{array}{r}7 \cdot 12 \\
\pm 0.48\end{array}$ & $\begin{array}{r}4.58 \\
\pm 0.33\end{array}$ & $\begin{array}{r}2.54 \\
\pm 0.17\end{array}$ \\
\hline $\begin{array}{l}\text { Patients 6-9 } \\
\text { Mean } \\
\pm \text { SD }\end{array}$ & $\begin{array}{r}11 \cdot 70 \\
\pm 1.03\end{array}$ & $\begin{array}{r}6.48 \\
\pm 0.96\end{array}$ & $\begin{array}{r}12 \cdot 50 \\
\pm 1 \cdot 16\end{array}$ & $\begin{array}{r}7 \cdot 53 \\
\pm 0.87\end{array}$ & $\begin{array}{r}6.92 \\
\pm 1.07\end{array}$ & $\begin{array}{r}4.98 \\
\pm 0.41\end{array}$ & $\begin{array}{r}0.63 \\
\pm 0.39\end{array}$ & $\begin{array}{r}0.01130 \\
\pm 0.00168\end{array}$ & $\begin{array}{r}7 \cdot 45 \\
\pm 1 \cdot 11\end{array}$ & $\begin{array}{r}3.65 \\
\pm 0.89\end{array}$ & $\begin{array}{r}3.80 \\
\pm 1.81\end{array}$ \\
\hline $\begin{array}{l}\mathbf{t} \\
\mathbf{P}\end{array}$ & $\begin{aligned} & 0.585 \\
> & 0.50\end{aligned}$ & $\begin{aligned} & 0.009 \\
> & 0.99\end{aligned}$ & $\begin{aligned} & 0.541 \\
> & 0.5\end{aligned}$ & $\begin{aligned} & 0.213 \\
> & 0.8\end{aligned}$ & $\begin{aligned} & 0.035 \\
> & 0.95\end{aligned}$ & $\begin{aligned} & 1.037 \\
> & 0.3\end{aligned}$ & $\begin{aligned} & 0.415 \\
> & 0.6\end{aligned}$ & $>0.2^{1 \cdot 123}$ & $\begin{aligned} & 0.607 \\
> & 0.5\end{aligned}$ & $\begin{aligned} & 2.193 \\
> & 0.05\end{aligned}$ & $\begin{aligned} & 1.580 \\
> & 0.1\end{aligned}$ \\
\hline
\end{tabular}

Table II Plasma calcium and protein fractions and $K_{\text {CaProt }}$ values for patients with hypercalcaemia ${ }^{1}$

${ }^{1}$ In patients 1 to 5 the diagnosis was primary hyperparathyroidism, and in patients 6 to 9 the hypercalcaemia was due to carcinomatosis. The comparison of these two groups by Students' $t$ index and the significance $P$ of the $t$ value is shown.

\begin{tabular}{|c|c|c|c|c|c|c|c|c|c|c|c|}
\hline \multirow[t]{2}{*}{ Patient } & \multicolumn{2}{|c|}{$\begin{array}{l}\text { Calcium } \\
(\mathrm{mg} / 100 \mathrm{ml} \text { plasma })\end{array}$} & \multicolumn{5}{|c|}{ Calcium ( $\mathrm{mg}$ per $100 \mathrm{ml}$ plasma water) } & \multirow[t]{2}{*}{$K_{\text {CaProt }}$} & \multicolumn{3}{|c|}{ Protein (g per $100 \mathrm{ml}$ plasma) } \\
\hline & Total & Ionized & Total & $\begin{array}{l}\text { Ultra- } \\
\text { filtrable }\end{array}$ & Ionized & $\begin{array}{l}\text { Protein- } \\
\text { bound }\end{array}$ & Complexed & & Total & Albumin & Gobulin \\
\hline $\begin{array}{l}10 \\
11 \\
12 \\
13 \\
14 \\
15 \\
16 \\
17 \\
18 \\
19 \\
20 \\
21\end{array}$ & $\begin{array}{l}8 \cdot 4 \\
8 \cdot 1 \\
8 \cdot 5 \\
8 \cdot 4 \\
6 \cdot 9 \\
8 \cdot 4 \\
8 \cdot 5 \\
8 \cdot 3 \\
7 \cdot 9 \\
7 \cdot 5 \\
8 \cdot 2 \\
8 \cdot 2\end{array}$ & $\begin{array}{l}5 \cdot 1 \\
4 \cdot 5 \\
4 \cdot 1 \\
5 \cdot 2 \\
4 \cdot 2 \\
4 \cdot 7 \\
4 \cdot 5 \\
4 \cdot 2 \\
4 \cdot 3 \\
3 \cdot 7 \\
3 \cdot 3 \\
4 \cdot 9\end{array}$ & $\begin{array}{l}8.9 \\
8 \cdot 6 \\
9 \cdot 0 \\
8 \cdot 9 \\
7 \cdot 3 \\
8 \cdot 9 \\
9 \cdot 0 \\
8 \cdot 9 \\
8 \cdot 4 \\
8 \cdot 0 \\
8 \cdot 7 \\
8 \cdot 7\end{array}$ & $\begin{array}{l}6 \cdot 5 \\
5 \cdot 5 \\
6 \cdot 2 \\
5 \cdot 6 \\
4 \cdot 5 \\
5 \cdot 2 \\
5 \cdot 9 \\
5 \cdot 1 \\
5 \cdot 0 \\
5 \cdot 4 \\
4 \cdot 1 \\
5 \cdot 5\end{array}$ & $\begin{array}{l}5 \cdot 5 \\
4 \cdot 8 \\
4 \cdot 4 \\
5 \cdot 5 \\
4 \cdot 5 \\
5 \cdot 0 \\
4 \cdot 8 \\
4 \cdot 4 \\
4 \cdot 5 \\
4 \cdot 0 \\
3 \cdot 5 \\
5 \cdot 2\end{array}$ & $\begin{array}{l}2.4 \\
3 \cdot 1 \\
2.8 \\
3.3 \\
2.8 \\
3.7 \\
3 \cdot 1 \\
3.8 \\
3.4 \\
2.6 \\
4.6 \\
3.2\end{array}$ & $\begin{array}{l}1.0 \\
0.7 \\
1.8 \\
0.1 \\
0.0 \\
0.2 \\
1.1 \\
0.7 \\
0.5 \\
1.4 \\
0.6 \\
0.3\end{array}$ & $\begin{array}{l}0.01843 \\
0.01160 \\
0.01201 \\
0.01351 \\
0.00995 \\
0.00984 \\
0.00994 \\
0.00935 \\
0.00946 \\
0.01324 \\
0.00544 \\
0.01099\end{array}$ & $\begin{array}{l}6 \cdot 8 \\
6 \cdot 8 \\
6 \cdot 4 \\
6 \cdot 9 \\
6 \cdot 1 \\
6 \cdot 6 \\
5 \cdot 6 \\
6 \cdot 9 \\
6 \cdot 1 \\
7 \cdot 3 \\
6 \cdot 4 \\
5 \cdot 9\end{array}$ & $\begin{array}{l}3 \cdot 7 \\
4 \cdot 3 \\
4 \cdot 6 \\
4 \cdot 6 \\
3 \cdot 4 \\
3 \cdot 9 \\
3 \cdot 5 \\
4 \cdot 3 \\
3 \cdot 9 \\
3 \cdot 7 \\
3 \cdot 3 \\
3 \cdot 8\end{array}$ & $\begin{array}{l}3 \cdot 1 \\
2 \cdot 5 \\
1 \cdot 8 \\
2 \cdot 3 \\
2 \cdot 7 \\
2 \cdot 7 \\
2 \cdot 1 \\
2 \cdot 6 \\
2 \cdot 3 \\
3 \cdot 6 \\
3 \cdot 1 \\
2 \cdot 1\end{array}$ \\
\hline $\begin{array}{l}\text { Mean } \\
\pm \text { SD }\end{array}$ & $\begin{array}{r}8 \cdot 11 \\
\pm 0.48\end{array}$ & $\begin{array}{r}4.39 \\
\pm 0.55\end{array}$ & $\begin{array}{r}8.60 \\
\pm 0.50\end{array}$ & $\begin{array}{r}5.36 \\
\pm 0.66\end{array}$ & $\begin{array}{r}4.66 \\
\pm 0.59\end{array}$ & $\begin{array}{r}3.24 \\
\pm 0.60\end{array}$ & $\begin{array}{r}0.70 \\
\pm 0.54\end{array}$ & $\begin{array}{r}0.01115 \\
\pm 0.00312\end{array}$ & $\begin{array}{r}6.48 \\
\pm 0.49\end{array}$ & $\begin{array}{r}3.92 \\
\pm 0.44\end{array}$ & $\begin{array}{r}2.58 \\
\pm 0.51\end{array}$ \\
\hline
\end{tabular}

Table III Plasma calcium and protein fractions and $K_{C u P r o t}$ values for patients with hypocalcaemia due to various non-renal causes ${ }^{1}$

In patient 11 the diagnosis was postsurgical hypoparathyroidism. 


\begin{tabular}{|c|c|c|c|c|c|c|c|c|c|c|c|c|}
\hline \multirow[t]{2}{*}{ Patient } & \multicolumn{2}{|c|}{$\begin{array}{l}\text { Calcium } \\
(\mathrm{mg} / 100 \mathrm{ml} \text { Plasma })\end{array}$} & \multicolumn{5}{|c|}{ Calcium (mg per $100 \mathrm{ml}$ plasma water) } & \multirow[t]{2}{*}{$K$ CaProt } & \multicolumn{3}{|c|}{ Protein ( $g$ per $100 \mathrm{ml}$ plasma) } & \multirow{2}{*}{$\begin{array}{l}\text { Blood } \\
\text { Urea } \\
\text { (mg per } \\
700 \mathrm{ml})\end{array}$} \\
\hline & Total & Ionized & Total & $\begin{array}{l}\text { Ultra- } \\
\text { filtrable }\end{array}$ & Ionized & $\begin{array}{l}\text { Protein- } \\
\text { bound }\end{array}$ & Complexed & & Total & Albumin & Globulin & \\
\hline 22 & $6 \cdot 5$ & $3 \cdot 1$ & 6.9 & $3 \cdot 8$ & $3 \cdot 3$ & $3 \cdot 1$ & 0.5 & 0.00718 & 6.4 & $3 \cdot 7$ & $2 \cdot 7$ & 110 \\
\hline 23 & $8 \cdot 5$ & $4 \cdot 4$ & $9 \cdot 1$ & 6.0 & $4 \cdot 7$ & $3 \cdot 1$ & $1 \cdot 3$ & 0.01200 & $7 \cdot 2$ & $4 \cdot 0$ & $3 \cdot 2$ & 156 \\
\hline 24 & $9 \cdot 0$ & 4.9 & $9 \cdot 6$ & $5 \cdot 7$ & $5 \cdot 2$ & 3.9 & 0.5 & 0.00956 & $6 \cdot 7$ & $3 \cdot 7$ & $3 \cdot 0$ & 164 \\
\hline 25 & $8 \cdot 3$ & $4 \cdot 7$ & $8 \cdot 8$ & $5 \cdot 9$ & $5 \cdot 0$ & 2.9 & 0.9 & 0.01414 & $7 \cdot 3$ & $4 \cdot 7$ & $2 \cdot 6$ & 144 \\
\hline 26 & $8 \cdot 5$ & $4 \cdot 0$ & $9 \cdot 1$ & $4 \cdot 9$ & $4 \cdot 3$ & $4 \cdot 2$ & 0.6 & 0.00986 & $8 \cdot 3$ & $4 \cdot 8$ & $3 \cdot 5$ & 86 \\
\hline 27 & $8 \cdot 1$ & $4 \cdot 6$ & 8.6 & $5 \cdot 0$ & 4.9 & $3 \cdot 6$ & $0 \cdot 1$ & 0.00980 & 6.5 & $4 \cdot 4$ & $2 \cdot 1$ & 300 \\
\hline 28 & $7 \cdot 6$ & $4 \cdot 0$ & $8 \cdot 2$ & $4 \cdot 7$ & $4 \cdot 3$ & $3 \cdot 5$ & 0.4 & 0.01087 & $8 \cdot 0$ & $4 \cdot 4$ & $3 \cdot 6$ & 260 \\
\hline 29 & 4.9 & $2 \cdot 7$ & $5 \cdot 2$ & $3 \cdot 1$ & $2 \cdot 8$ & $2 \cdot 1$ & $0 \cdot 3$ & 0.00853 & $5 \cdot 5$ & $3 \cdot 5$ & $2 \cdot 0$ & 54 \\
\hline 30 & $7 \cdot 5$ & $4 \cdot 2$ & $8 \cdot 1$ & $4 \cdot 8$ & $4 \cdot 5$ & $3 \cdot 3$ & $0 \cdot 3$ & 0.01289 & $7 \cdot 8$ & $5 \cdot 5$ & $2 \cdot 3$ & 190 \\
\hline $\begin{array}{l}\text { Mean } \\
+ \text { SD }\end{array}$ & $\begin{array}{r}7.66 \\
\div 1.26\end{array}$ & $\begin{array}{r}4.07 \\
+0.73\end{array}$ & $\begin{array}{r}8.16 \\
-1.36\end{array}$ & $\begin{array}{r}4.88 \\
-0.96\end{array}$ & $\begin{array}{r}4.32 \\
+0.80\end{array}$ & $\begin{array}{r}3.30 \\
+0.61\end{array}$ & $\begin{array}{r}0.54 \\
+0.36\end{array}$ & $\begin{array}{r}0.01054 \\
+0.00218\end{array}$ & $\begin{array}{r}7.08 \\
-0.89\end{array}$ & $\begin{array}{r}4.30 \\
-0.64\end{array}$ & $\begin{array}{r}2.78 \\
+0.59\end{array}$ & - \\
\hline$\pm \mathbf{S D}$ & $=1 \cdot 26$ & \pm 0.73 & $=1.36$ & $=0.96$ & \pm 0.80 & $\mp 0.61$ & \pm 0.36 & \pm 0.00218 & \pm 0.89 & $=0.64$ & \pm 0.59 & - \\
\hline
\end{tabular}

Table IV Plasma calcium and protein fractions, blood urea, and $K_{C a P r o t}$ values for patients with chronic renal failure

The individual results of plasma calcium fractions and $\mathrm{K}_{\mathrm{CaProt}}$ values together with the mean and SD values for the groups of patients with hypercalcaemia, non-renal hypocalcaemia, and renal hypocalcaemia are detailed in Tables II, III, and IV respectively.

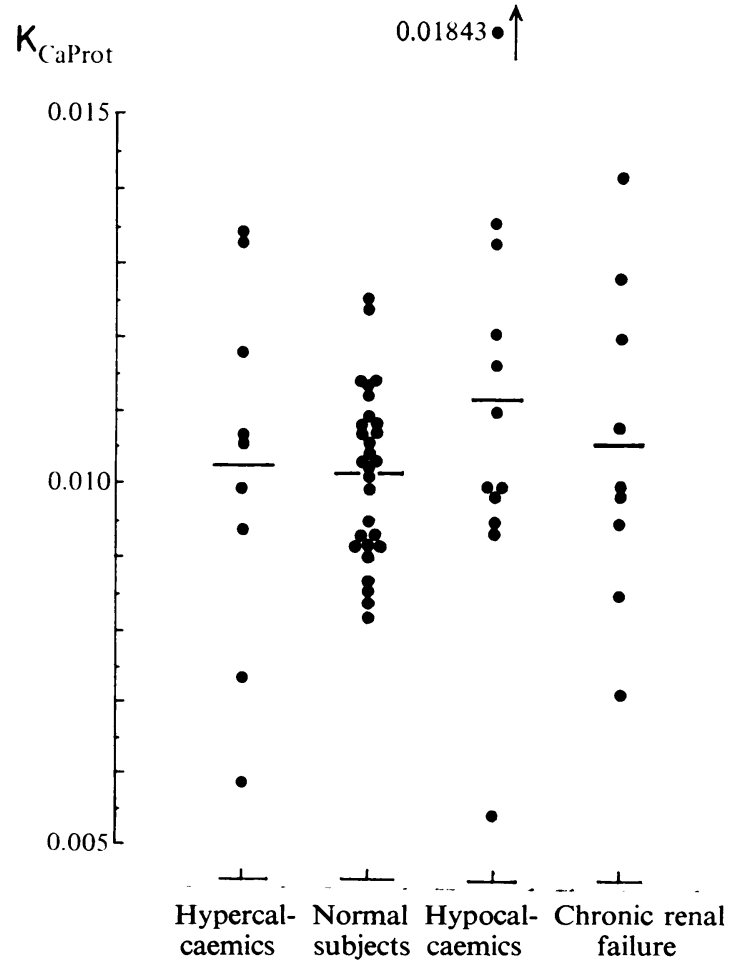

Fig. 1 Calcium proteinate dissociation constant for normal subjects and the groups of patients studied (mean and individual values).

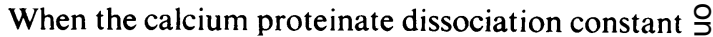
of the normal subjects was compared with those of the individual patient groups by Student's $t$ index there was no significant difference between the $\mathcal{\Phi}_{0}$ normal subjects and the patient with chronic renal failure $(P>0.7)$, the patients with hypercalcaemia $\underset{\Phi}{ }$ $(\mathrm{P}>0.9)$, or the patients with hypocalcaemia $(\mathrm{P}>0 \cdot 3)$. The individual results, however, showed a wider 'scatter' in the groups of patients when compared with the normal subjects (Figure 1). There was no significant difference between the calcium proteinate dissociation constant for the patients with hyperparathyroidism and those with hypercalcaemia due to other causes $(\mathrm{P}>0 \cdot 2)$. The mean and SD values for the acid-base status of the normal subjects and groups of patients are shown in Table $\mathrm{V}$. In the group of patients with renal hypocalcaemia there was a metabolic acidosis as reflected by significant differences from normal for both blood $p \mathrm{H}$ and bicarbonate values; the effect of $p \mathrm{H}$ on the dissociation constant is allowed for in the formula for calculating total cation-binding proteins.

In order to investigate the relationship between the total plasma calcium concentration and the ultrafiltrable, ionized, protein-bound, and com- I plexed fractions over the whole range studied the individual results for these fractions for all the $N$ patients and normal subjects were plotted as shown $N$ in Figures 2, 3, 4, and 5, and both the regression and correlation coefficients calculated. There was a highly significant correlation between the total and ultrafiltrable, between the total and ionized, and

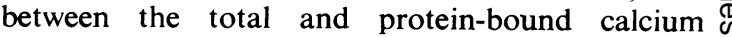
fractions, but no correlation between the total and complexed calcium fraction for the whole sample studied. When the normal subjects and groups of patients were considered individually there was also a significant correlation between the total and ultra- 


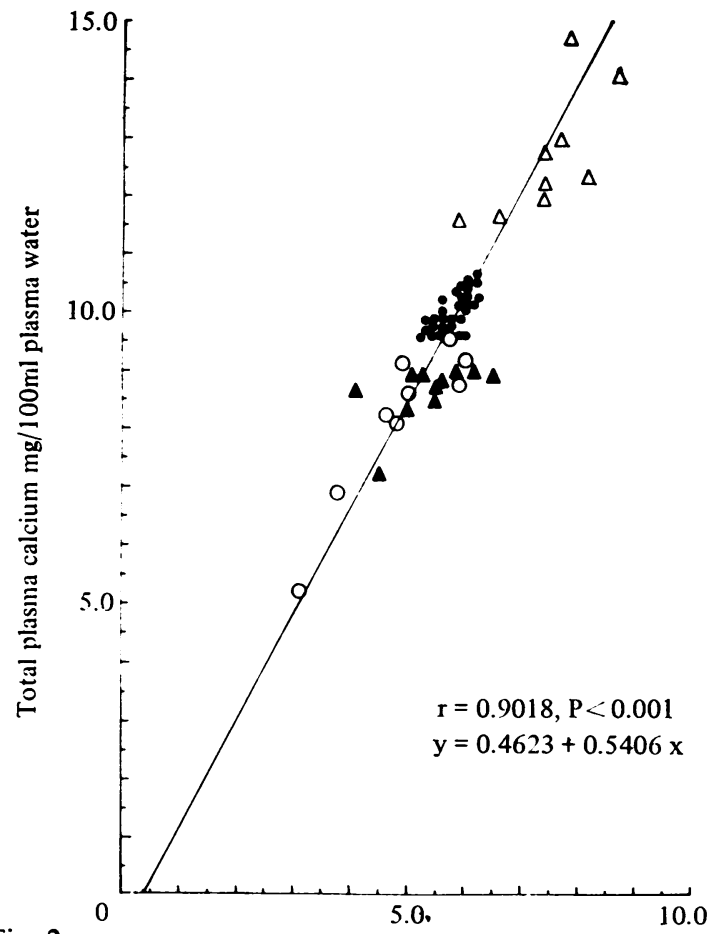

Fig. 2

Ultrafiltrable calcium $\mathrm{mg} / 100 \mathrm{ml}$ ultrafiltrate

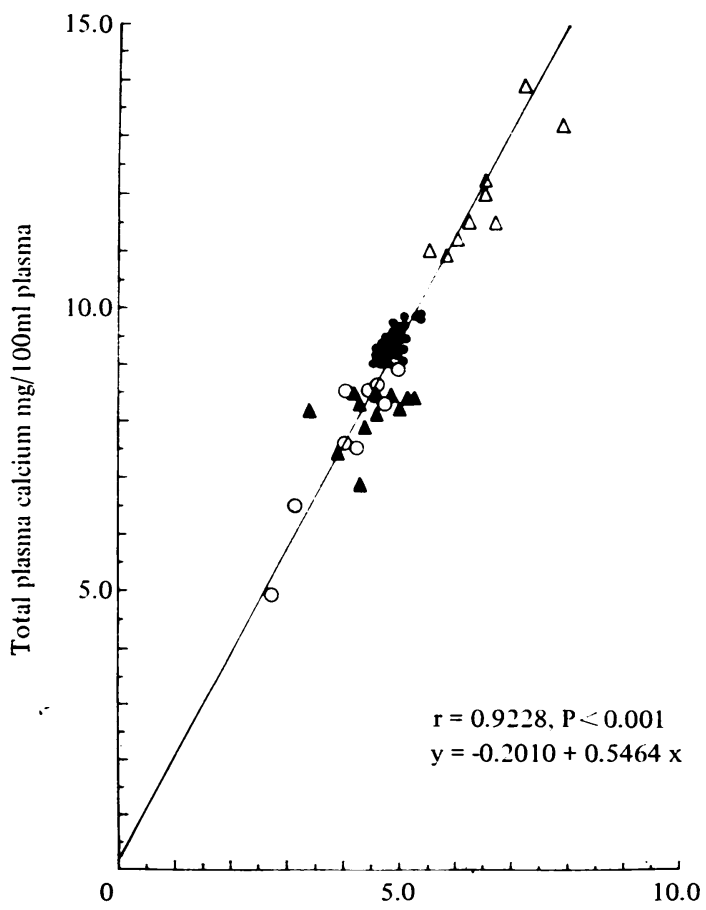

Fig. 3

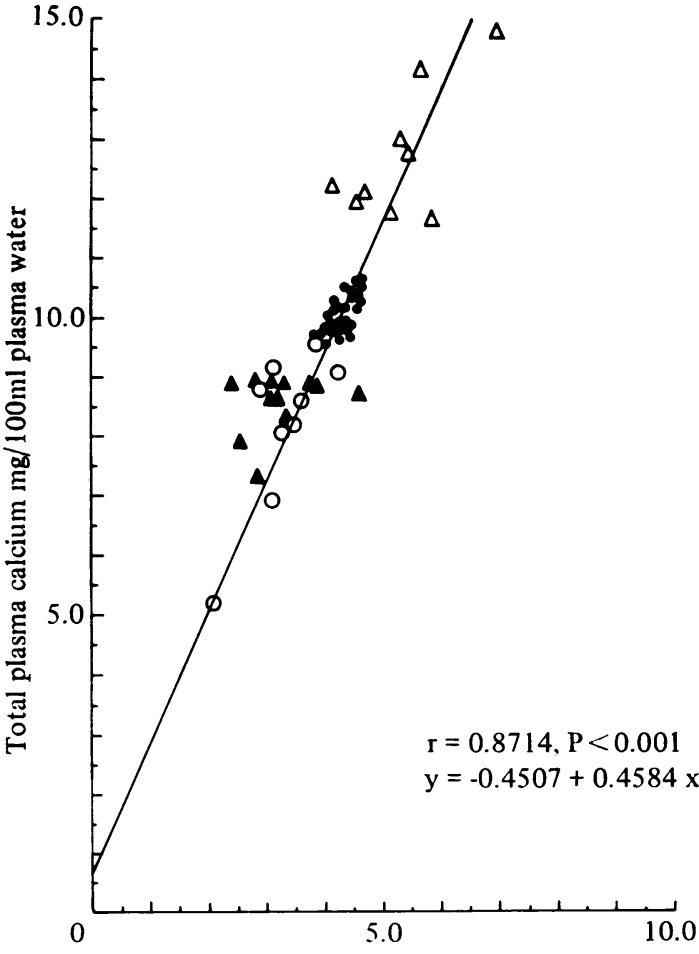

Protein-bound calcium $\mathrm{mg} / 100 \mathrm{ml}$ plasma water

Fig. 4

Fig. 2 Total versus ultrafiltrable calcium concentration for normal subjects $(\Theta)$, patients with hypercalcaemia $(\triangle)$, patients with renal hypocalcaemia $(O)$, and with non-renal hypocalcaemia $(\mathbf{\Delta})$.

Fig. 3 Total versus ionized calcium concentration, in plasma, for normal subjects $(\Theta)$, patients with hypercalcaemia $(\triangle)$, patients with renal hypocalcaemia $(\bigcirc)$, and with non-renal hypocalcaemia $(\Delta)$.

Fig. 4 Total versus protein-bound calcium concentration for normal subjects $(\bigcirc)$, patients with hypercalcaemia $(\triangle)$, patients with renal hypocalcaemia $(O)$, and with non-renal hypocalcaemia $(\boldsymbol{\Delta})$. 


\begin{tabular}{|c|c|c|c|c|}
\hline \multirow[t]{2}{*}{ Group } & & \multicolumn{2}{|l|}{ Blood } & \multirow{2}{*}{$\frac{\text { Plasma }}{\text { Bicarbonate }(\text { m-equiv/l) }}$} \\
\hline & & $p H$ & $\mathrm{PCO}_{2}(\mathrm{~mm} \mathrm{Hg})$ & \\
\hline Normal subjects $n=29$ & $\begin{array}{l}\text { Mean } \\
\pm \mathrm{SD}\end{array}$ & $\begin{array}{r}7.33 \\
\pm 0.03\end{array}$ & $\begin{array}{r}43 \cdot 8 \\
\pm \quad 4 \cdot 1\end{array}$ & $\begin{array}{r}25 \cdot 3 \\
+\quad 3 \cdot 5\end{array}$ \\
\hline Hypercalcaemics $n=9$ & $\begin{array}{l}\text { Mean } \\
\pm S D\end{array}$ & $\begin{array}{r}7 \cdot 35 \\
\pm 0.08\end{array}$ & $\begin{array}{r}41 \cdot 3 \\
\pm \quad 3 \cdot 6\end{array}$ & $\begin{array}{r}24 \cdot 2 \\
\pm 4 \cdot 2\end{array}$ \\
\hline Non-renal hypocalcaemia $n=12$ & $\begin{array}{l}\text { Mean } \\
\pm S D\end{array}$ & $\begin{array}{r}7 \cdot 37 \\
\pm 0.05\end{array}$ & $\begin{array}{r}41.8 \\
\pm 4.6\end{array}$ & $\begin{array}{r}23 \cdot 3 \\
+\quad 3 \cdot 4\end{array}$ \\
\hline Renal hypocalcaemia $n=9$ & $\begin{array}{l}\text { Mean } \\
\pm \mathrm{SD}\end{array}$ & $\begin{array}{r}7.29 \\
\pm 0.05\end{array}$ & $\begin{array}{r}41 \cdot 6 \\
\pm \quad 8.2\end{array}$ & $\begin{array}{r}20 \cdot 8 \\
+\quad 4 \cdot 0\end{array}$ \\
\hline $\begin{array}{l}\text { Normals v } \\
\text { hypercalcaemics }\end{array}$ & $\begin{array}{l}\mathrm{t} \\
\mathrm{P}\end{array}$ & $\begin{aligned} & 0.41 \\
> & 0.6\end{aligned}$ & $\begin{aligned} & 1.46 \\
> & 0.1\end{aligned}$ & $\begin{aligned} & 0.66 \\
> & 0.5\end{aligned}$ \\
\hline $\begin{array}{l}\text { Normals } \mathrm{v} \\
\text { non-renal hypocalcaemia }\end{array}$ & $\begin{array}{l}t \\
P\end{array}$ & $\begin{array}{r}2.13 \\
<0.05\end{array}$ & $\begin{aligned} & 1.20 \\
> & 0.2\end{aligned}$ & $\begin{array}{ll} & 1.47 \\
> & 0.1\end{array}$ \\
\hline $\begin{array}{l}\text { Normals } \mathrm{v} \\
\text { renal hypocalcaemia }\end{array}$ & $\begin{array}{l}\mathrm{t} \\
\mathbf{P}\end{array}$ & $\begin{array}{r}2.97 \\
<0.01\end{array}$ & $\begin{aligned} & 0.83 \\
> & 0.4\end{aligned}$ & $\begin{array}{r}2.87 \\
<0.01\end{array}$ \\
\hline
\end{tabular}

Table $\mathrm{V}$ Acid-base status of groups of patients and normal subjects ${ }^{1}$

${ }^{1}$ Comparison of results by Student's $t$ index and significance $P$ of the $t$ values.

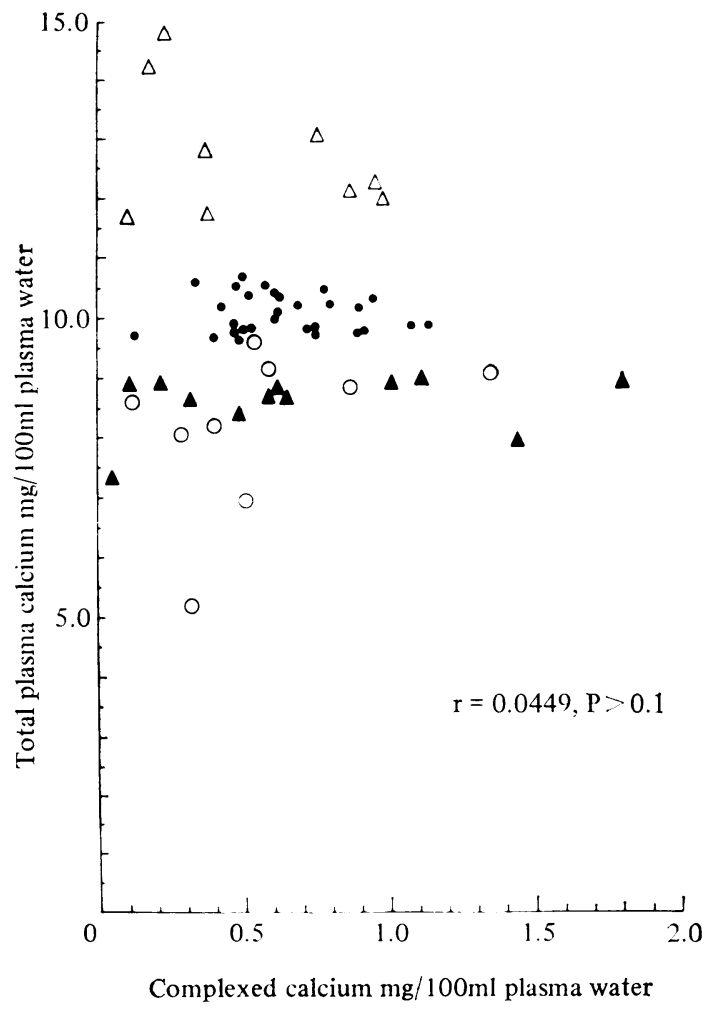

Fig. 5 Total versus complexed calcium concentration for normal subjects $(\Theta)$, patients with hypercalcaemia $(\triangle)$, patients with renal hypocalcaemia $(\bigcirc)$, and with non-renal hypocalcaemia $(\mathbf{\Delta})$. filtrable, between the total and ionized, and between the total and protein-bound calcium fractions in the normal subjects ( $P$ values $<0.001$ ), and the group of patients with renal hypocalcaemia and the $\vec{\theta}$ group with hypercalcaemia ( $P$ values $<0.05)$. In patients with non-renal hypocalcaemia there was no correlation between either the total and ultrafiltrable, the total and ionized, or the total and protein-bound calcium fractions ( $P$ values $>0 \cdot 1$ ). It can be seen in Figure 2 that the 'fit' of points on the regression line $\stackrel{\mathbb{Q}}{\mathbb{2}}$ for the concentration of the ultrafiltrable fraction $\vec{F}$ was not as good as with the ionized fraction (Figure 3). This lack of 'fit' is probably due to the fact that the concentration of the ultrafiltrable fraction is dependent not only on ionized calcium but also on complexed calcium, which will depend on the concentration of complexing factors in the plasma. In view of the significant correlation between the total and the ultrafiltrable, and between the total and the ionized plasma calcium fractions

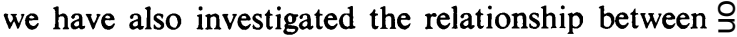
the ultrafiltrable and the ionized plasma calcium $D$ fractions (Figure 6). There was a significant correlation between these two fractions for the sample $N$ as a whole $(P<0.001)$, and for the normal subjects, patients with hypercalcaemia, renal hypocalcaemia 0 ( $P$ values $<0.001$ ), and non-renal hypocalcaemia $\omega$ $(P<0.05)$ when treated as separate groups. The $95 \%$ O confidence limits for a predicted value of ionized 0 calcium $(Y)$ given a measured concentration of the ultrafiltrable fraction $(X)$ are shown in Figure 6.

\section{Discussion}

In this study we have shown that the calcium-binding $\stackrel{\mathbb{Q}}{\stackrel{Q}{2}}$ 


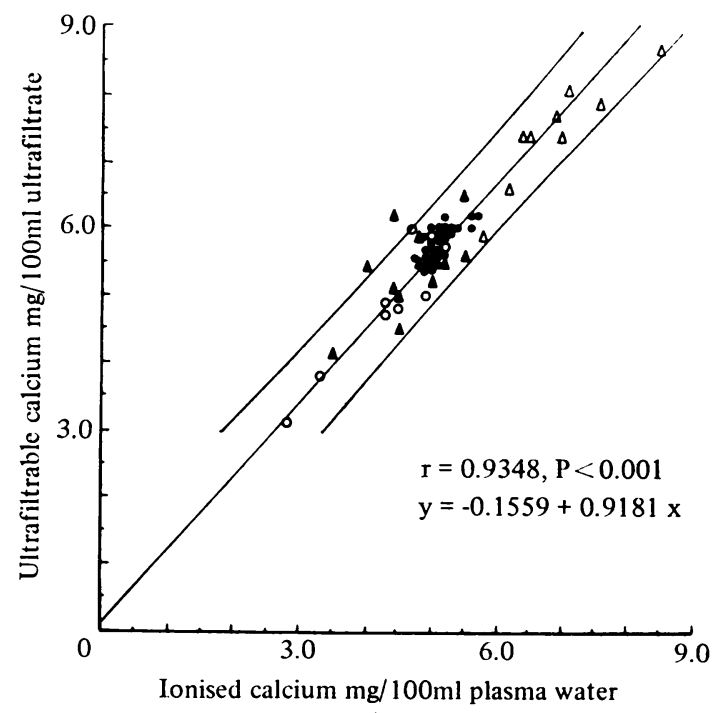

Fig. 6 Ultrafiltrable versus ionized calcium concentration for normal subjects $(\bullet)$, for patients with hypercalcaemia $(\triangle)$, patients with renal hypocalcaemia $(\bigcirc)$, and with non-renal hypocalcaemia $(\mathbf{\Delta})$. Also shown are the $95 \%$ confidence limits for a predicted value of ionized calcium given a measured concentration of the ultrafiltrable fraction.

affinity of the plasma proteins as determined by calcium proteinate dissociation constant ( $\left.\mathrm{K}_{\mathrm{CaProt}}\right)$, calculated from data collected under physiological conditions of $p \mathrm{H}$ and temperature, did not differ significantly between groups of patients with hypercalcaemia, those with hypocalcaemia due either to renal or non-renal causes, and normal subjects. It has also been demonstrated that for the whole sample studied, over a wide range of total calcium concentration there was a significant correlation between the total plasma calcium concentration and the ultrafiltrable, ionized, and protein-bound fractions. This is to be expected when plasma protein concentrations are normal. We have also found that a significant correlation existed between these variables when the normal subjects and the groups of patients with renal hypocalcaemia and hypercalcaemia were considered separately. However, in the group of patients with non-renal hypocalcaemia, although the calcium-binding affinity of the plasma proteins did not differ significantly from either the normal subjects or the other groups of patients, we were unable to demonstrate any correlation between the total plasma calcium concentration and the concentration of any of the plasma calcium fractions, which implies an abnormality of either protein concentrations or complexing factors (vide infra). In neither the whole sample studied nor in any of the individual groups was there any correlation between the total plasma calcium concentration and the complexed calcium fraction. This lack of correlation was presumably accounted for, first, by the low concentration of complexed calcium which exists in plasma and the fact that it was derived from the difference between the ultrafiltrable and ionized calcium fractions so that the precision of the estimation is low; secondly, by the fact that it is dependent on the concentration of the complexing factors present in plasma which may vary considerably. The results suggest that (1) the estimation of ultrafiltrable fraction alone is a reliable index of the concentration of the ionized calcium concentration, and (2) the concentrations of the ultrafiltrable, ionized, and protein-bound calcium concentrations can probably be predicted from the total plasma calcium concentration, unless there are marked changes in total plasma protein concentration. These observations are in accordance with some, but by no means all, of the earlier reports in the literature.

Reports in the literature of the state of the plasma calcium fractions and calcium-binding affinity of the plasma proteins in patients with hypercalcaemia have been conflicting. In a study of 27 patients with hypercalcaemia, most of whom had normal total proteins, and which included 13 patients with primary hyperparathyroidism, Walser (1962) reported that 'the percentage ionization of plasma calcium was normal' and thus any increase in total calcium concentration would be reflected by proportional increases in the ultrafiltrable, ionized, and protein-bound calcium fractions. These findings are consistent with those reported here and those of other workers who have reported similar studies (Fowler, Fone, and Cooke, 1961; Hodgkinson and Edwards, 1963; Kotzaurek, 1967; Robertson and Peacock, 1968). Walser (1962) also reported that the calcium-binding affinity of the plasma proteins in patients with hypercalcaemia, whether the latter was due to hyperparathyroidism or various other causes, was unaltered when compared with normal subjects. Similar results were obtained by Dale and Kellerman (1967), who concluded that the determination of the calcium affinity of plasma proteins was of no special value in the differential diagnosis of hypercalcaemia. This view is consistent with the findings reported here both from the results of the calculation of protein-bound calcium fraction and determination of the calcium proteinate dissociation constant.

Other workers, however, have reported that in patients with hypercalcaemia due to hyperpara- 
thyroidism there was a dispropoitionate increase in either the ionized or ultrafiltrable calcium fraction when compared with patients with hypercalcaemia due to other causes.

Hellstrom (1953) mentioned three patients with hyperparathyroidism with normal total plasma calcium concentrations in whom there was an increase in the ultrafiltrable fraction, which returned to normal after operation; the plasma protein concentrations in these three patients were not mentioned. In a study of 17 patients with hyperparathyroidism (including four of the cases reported by Fanconi and Rose, 1958) Lloyd and Rose (1958) reported that the ionized calcium concentration preoperatively was above normal in all but one case. The one exception they considered was in a temporary remission as at operation the parathyroid adenoma was shown to contain a focus of degeneration. After removal of the parathyroid adenoma from those patients with a high preoperative ionized calcium concentration, Lloyd and Rose reported that the postoperative fall in the ionized calcium fraction was much greater in relation to the total calcium than could be accounted for by any change in protein concentration. They inferred from these studies that parathyroid hormone reduced the ability of the plasma proteins to bind calcium. This view was supported in subsequent studies by Lloyd et al (1962) who reported that the reduction of the ability of the plasma proteins to bind calcium was due to the hyperparathyroidism itself and not to the hypercalcaemia, as no reduction in calcium-binding was seen in patients with hypercalcaemia due to other causes.

In a study of three patients with hyperparathyroidism Breen and Freeman (1961a) reported that the concentrations of ultrafiltrable calcium were increased and they considered that these findings were in agreement with those of Rose and his colleagues (Fanconi and Rose, 1958; Lloyd and Rose, 1958). However, in these studies they demonstrated that the amount of calcium bound per $g$ of protein, in these three patients, was significantly higher than in normal subjects. In subsequent studies on experimental animals (dogs and rats) with parathyroidextract-induced hypercalcaemia Breen and Freeman (1961b) reported that as the total calcium concentration increased, there was a proportionate increase in the ultrafiltrable and protein-bound calcium concentration. Breen and Freeman concluded from these later studies that the concentration of the protein-bound calcium was a function of the total concentration of calcium and protein, and that parathyroid hormone only influenced the distribution of plasma calcium indirectly by regulating its total concentration. The conclusion is consistent with the findings reported here and those of other workers which have already been discussed.

In patients with non-renal hypocalcaemia the position with respect to plasma calcium fractions and the calcium-binding affinity of the plasma proteins has been equally conflicting, although there have been fewer studies. Terepka, Toribara, and Dewey (1958) reported that in patients with hypocalcaemia and normal total plasma protein concentration the percentage ultrafiltrable calcium fraction was normal while in hypocalcaemic-hypoproteinaemic patients the percentage ultrafiltrable fraction was increased. These findings have been confirmed by other workers (Fowler et al, 1961; Kotzaurek, 1967), and would suggest that the reduction of total protein concentration had produced the expected reduction of protein calcium-binding sites. This view is supported by the studies reported here in that we have shown that the calcium-binding affinity of the plasma proteins in the non-renal hypocalcaemic patients did not differ significantly from that obtained in either the normal subjects or in the patients in the other groups. In this group we were, however, unable to show any correlation between the total plasma calcium concentration and the concentration of any of the calcium fractions. This implies an abnormality either of protein concentration or of $p \mathrm{H}$. The blood $p \mathrm{H}$ in this group was significantly higher than in the normal subjects (Table V) which may account in part at least for our findings. However, in the group of non-renal hypocalcaemic patients the total plasma protein concentration was significantly lower $(\mathrm{P}<0.001)$ than in the normal subjects, and when the protein effect was eliminated by comparing the ionized and ultrafiltrable concentrations in this group there was a significant correlation $(\mathrm{P}<0.05)$ between these two fractions. In the other two groups of patients the total plasma protein concentration did not differ significantly from that in the normal subjects ( $P$ values $>0.5$ and $>0.2$ for the groups of patients with hypercalcaemia and chronic renal failure respectively).

In hypocalcaemic patients with chronic renal failure tetanic episodes are extremely rare, and the absence of hypocalcaemic tetany in these patients is usually attributed to the concomitant acidosis. The 'protective' role of the acidosis has been attributed either to an increase in the concentration of the ionized calcium fraction by diminishing the protein-bound fraction or to a specific effect of the hydrogen ion on neuromuscular irritability. In two of our patients with renal hypocalcaemia there was a marked reduction in both the total and the ionized plasma calcium fractions but neither had tetany. It has been established that over the whole $p \mathrm{H}$ range there is a considerable variation in the amount of 
calcium that is bound to the plasma proteins (Prasad and Flink, 1957; Toribara, Terepka, and Dewey, 1957; Loken et al, 1960). However, from examination of the data of these authors it can be seen that variations in blood $p \mathrm{H}$ over the range of $7 \cdot 2$ to $7 \cdot 6 p \mathrm{H}$ units are associated with relatively small changes in the protein-bound calcium fraction. Over that range Walser (1962) calculated from the previously reported data of other authors that for an 0.3 unit change in blood $p \mathrm{H}$ there was an associated increase in the ultrafiltrable calcium concentration of approximately $6 \%$ of total plasma calcium. It is unlikely therefore that the variations in acid-base status which occur in disease states significantly affect the state of the calcium fractions present in the plasma compartment. In the study reported here we have shown that in the patients with chronic renal failure who had a metabolic acidosis (Table V) the calciumbinding affinity of the plasma proteins was normal and that changes in the total plasma calcium concentration were reflected by proportional changes in the ultrafiltrable, ionized, and protein-bound calcium fractions.

In a study of 11 patients with chronic renal disease and hypocalcaemia, of whom none had tetany, Terepka et al (1958) reported that, in all except two, the percentage ultrafiltrable calcium fraction was increased. They regarded their findings as evidence of some fundamental alteration in the ability of the plasma proteins to bind calcium as a direct consequence of the metabolic disturbance of chronic renal failure rather than due to the alteration in hydrogen ion concentration accompanying the metabolic acidosis of that condition. In six patients with chronic renal failure Fanconi and Rose (1958) found values for the plasma ionized calcium concentration that were normal in four, above normal in one, and below in the other, but nevertheless they concluded that the absence of tetany in patients with chronic renal failure was due not only to the acidosis but also to some other factor which may be an increase in plasma magnesium concentration which they found in one case. Support for this view has been expressed recently by Lim, Jacob, and Khoo (1969). Hypermagnesaemia has classically been considered as a biochemical disturbance associated with chronic renal failure but in recent years this has been disputed (see Wills, 1968). We did not measure magnesium concentrations in the patients reported here, but in our own experience the values are usually within the normal range in both dialysed and undialysed patients with end-stage chronic renal failure (Hollinrake, Thomas, Wills, and Baillod, 1970; Wills and Moorhead, unpublished data).

Walser (1962) measured the ionized and ultra- filtrable calcium fractions in 25 patients with chronic renal failure and azotaemia, of whom all except one were hypocalcaemic. He reported that the concentration of the ionized calcium fraction showed a constant relationship to the total plasma calcium concentration, and there was no significant difference between those with severe and those with mild acidosis, nor did the results differ from those he obtained in normal subjects and in patients with hypercalcaemia due to various causes including hyperparathyroidism. Walser (1962) also reported that the calcium-binding affinity of the plasma proteins in azotaemic chronic renal failure did not significantly differ from that in normal subjects. The findings reported here confirm those of Walser (1962) and support the hypothesis that absence of tetany in hypocalcaemic uraemic patients is due to a specific effect of the increased hydrogen ion concentration on neuromuscular irritability and is not due to any alteration in the state of the plasma calcium fractions as a result of the acidotic state.

The results obtained in this study would suggest that in normoproteinaemic patients the concentrations of the individual plasma calcium fractions can be derived from an estimation of the total plasma calcium concentration. In order to equate the concentrations of the individual plasma calcium fractions the results should be expressed in the same unit and reported in terms of $\mathrm{mg}$ per $100 \mathrm{ml}$ plasma water. The formula used for the determination of plasma water content includes the total plasma protein concentration. In those patients with marked alterations in plasma proteins the present study would suggest that the concentration of the ionized calcium fraction cannot be derived from the total plasma calcium concentration but can be predicted from the estimation of the total calcium concentration in the plasma ultrafiltrate.

In conclusion, a study is reported of the estimation of plasma calcium fractions and the calcium-binding affinity of the plasma proteins in a sample which comprised 29 normal subjects, nine patients with hypercalcaemia (primary hyperparathyroidism 5 , and metastatic carcinoma 4), and 21 patients with hypocalcaemia, associated with chronic renal failure in nine patients and with various other causes in 12 patients.

Determination of the calcium proteinate dissociation from data measured under physiological conditions of $p \mathrm{H}$ and temperature showed that there was no significant difference in the calciumbinding affinity of plasma proteins when the separate groups of patients were compared with the normal subjects. When the normal subjects and patients were considered together as a single group there was a significant correlation between the total plasma 
calcium concentration and between the ultrafiltrable, ionized, and protein-bound calcium fractions. There was also a significant correlation between the ultrafiltrable and ionized plasma calcium fractions for the sample as a whole, and for the individual groups of normal subjects and patients. When normal subjects, patients with hypercalcaemia, and patients with renal hypocalcaemia were considered as separate groups there was a significant correlation between the total plasma calcium concentration and the ultrafiltrable, ionized, and protein-bound calcium fractions. In the patients with non-renal hypocalcaemia there was no significant correlation between the total plasma calcium concentration and any of the plasma calcium fractions. In this group of patients the mean total plasma protein concentration was significantly lower than in the normal subjects and it is postulated that this factor, in part at least, accounts for the lack of correlation.

We wish to thank the Endowment Fund of the Royal Free Hospital under whose financial support this work was undertaken. We also wish to thank our clinical colleagues for permission to study patients under their care. We are grateful to $\mathrm{Dr}$ G. K. McGowan for valuable discussions on the preparation of this manuscript.

\section{References}

Breen, M., and Freeman, S. (1961a). The use of high centrifugal force in separating plasma calcium into its protein-bound and protein-free fractions. Clin. chim. Acta, 6, 181-187.

Breen, M., and Freeman, S. (1961b). Plasma calcium distribution in relation to parathyroid function. Amer.J.Physiol., 200,341-344.

Dale, N. E., and Kellerman, G. M. (1967). The binding of calcium by the plasma proteins in hyperparathyroidism. Clin. Sci., 32, 433-442.

Ettori, J., and Scoggan, S. M. (1961). A determination of ionized calcium in serum by two-wavelength spectrophotometry of a metal indicator. Clin. chim. Acta, 6, 861-865.

Fanconi, A., and Rose, G. A. (1958). The ionized, complexed and protein-bound fractions of calcium in plasma. Quart. J. Med., 27, 463-494.

Fourman, P., and Royer, P. (1968). Calcium Metabolism and the Bone. Blackwell Scientific Publications, Oxford.

Fowler, D. I., Fone, D. J., and Cooke, W. T. (1961). The calcium fractions of plasma in hypercalcaemic conditions and in adult coeliac disease. Lancet, 2, 284-287.

Hellstrom, J. (1953). Observations regarding the prognosis and diagnosis of hyperparathyroidism. Cleveland clin. Quart., 20, 253-256.

Hodgkinson, A., and Edwards, N. A. (1963). Total and ultrafiltrable serum calcium in primary hyperparathyroidism. Brit. J. Urol., $35,445-456$.

Hollinrake, K., Thomas, P. K., Wills, M. R., and Baillod, R. A. (1970).
Observations on plasma magnesium levels in patients with 0 uremic neuropathy under treatment by periodic hemodialysis. Neurology (Minneap.), 20, 939-942.

Hopkins, T., Howard, J. E., and Eisenberg, H. (1952). Ultrafiltration studies on calcium and phosphorus in human serum. Bull. Johns Hopk. Hosp., 91, 1-21.

Kotzaurek, R. (1967). Uber Erfahrungen mit neueren Untersuchungsmethoden bei Mineralstoffwechselstudien. II. Ionisiertes $\frac{\sigma}{}$ Calcium im Blutserum. Arch. klin. Med. 213, 96-105.

Lewin, M. R., Wills, M. R., and Baron, D. N. (1969). Ultramicrofluorimetric determination of calcium in plasma.J. clin. Path., ڤ્ల 22, 222-225.

Lim, P., Jacob, E., and Khoo, O. T. (1969). Serum ionized calcium $\vec{\circ}$ level in chronic renal failure. Med. J. Aust., 1, 1172-1174.

Lloyd, H. M., and Rose, G. A. (1958). Ionised protein-bound, and $\overrightarrow{\vec{\omega}}$ complexed calcium in the plasma in primary hyperparathy- $\omega$ roidism. Lancet, 2, 1258-1261.

Lloyd, H. M., Rose, G. A., and Smeenk, D. (1962). The ability of plasma proteins to bind calcium in normal subjects, in patients with primary hyperparathyroidism, both pre- and postoperatively and in other hypercalcaemic conditions. Clin. Sci., 22, 353-362.

Loken, H. F., Havel, R. J., Gordan, G. S., and Whittington, S. L. (1960). Ultracentrifugal analysis of protein-bound and free $\sigma$ calcium in human serum. J. biol. Chem., 235, 3654-3658.

Lumb, G. A. (1963). Determination of ionic calcium in serum. Clin. chim. Acta, 8, 33-38.

McLean, F. C., Barnes, B. O., and Hastings, A. B. (1934). Influence of thyro-parathyroidectomy and of parathyroid hormone upon state of calcium in serum of the cat. Proc. Soc. exp. Biol. (N.Y.), $\mathbb{D}$ 32, 253-260. McLean, F. C., and Hastings, A. B. (1935). The state of calcium in
the fluids of the body. J. biol. Chem., 108, 285-322.

Natelson, S. (1951). Routine use of ultramicro methods in the clinical $\vec{\theta}$ laboratory. Amer.J. clin. Path., 21, 1153-1172.

Neuman, W. F., and Neuman, M. W. (1958). The Chemical Dynamics $\rightarrow$ of Bone Mineral. University of Chicago Press, Chicago.

Pedersen, K. O. (1969). Determination of calcium fractions of serum. I. The separation of protein-bound and protein-free fractions by means of a simplified ultrafiltration technique. Scand.J. clin. Lab. Invest., 24, 69-81.

Prasad, A. S., and Flink, E. B. (1957). Effect of carbon dioxide on concentration of calcium in an ultrafiltrate of serum obtained $\frac{2}{\mathbb{D}}$ by centrifugation. J. appl. Physiol., 10, 103-107.

Robertson, W. G., and Peacock, M. (1968). New techniques for the $\overrightarrow{\vec{F}}$ separation and measurement of the calcium fractions of normal human serum. Clin. chim. Acta, 20, 315-326.

Van Slyke, D. D., Hastings, A. B., Hiller, A., and Sendroy, J., Jr. (1928). Studies of gas and electrolyte equilibria in blood. XIV. The amount of alkali bound by serum albumin and globulin. J. biol. Chem., 79, 769-780.

Terepka, A. R., Toribara, T. Y., and Dewey, P. A. (1958). The ultrafiltrable calcium of human serum. II. Variations in disease $\bar{O}$ states and under experimental conditions. J. clin. Invest., 37, 87-98.

Toribara, T. Y., Terepka, A. R., and Dewey, P. A. (1957). The ultrafiltrable calcium of human serum. I. Ultrafiltration methods and normal values. J. clin. Invest., 36, 738-748.

Walser, M. (1962). The separate effects of hyperparathyroidism, $\frac{\mathrm{O}}{2}$ hypercalcemia of malignancy, renal failure and acidosis on the state of calcium, phosphate and other ions in plasma. J. clin. Invest., 41, 1454-1471.

Wills, M. R. (1968). Biochemical consequences of chronic renal failure: a review, $J$, clin, Path., 21, 541-554.

Wolfson, W. Q., Cohn, C., Calvary, E., and Ichiba, F. (1948). Studies in serum proteins: V. A rapid procedure for the estimation of $\mathrm{O}$ total protein, true albumin, total globulin, alpha globulin, $N$ beta globulin and gamma globulin in $1.0 \mathrm{ml}$ of serum. Amer. J. clin. Path., 18, 723-730. 